\title{
Anisotropy and Percolation Threshold in a Multifractal Support
}

\author{
L. S. Lucena, J. E. Freitas, G. Corso, and R. F. Soares \\ International Center for Complex Systems and Departamento de Física Teórica e Experimental, \\ Universidade Federal do Rio Grande do Norte, Campus Universitário 59078 970, Natal, RN, Brazil
}

Received on 16 May, 2003

\begin{abstract}
Recently a multifractal object, $Q_{m f}$, was proposed to allow the study of percolation properties in a multifractal support. The area and the number of neighbors of the blocks of $Q_{m f}$ show a non-trivial behavior. The value of the probability of occupation at the percolation threshold, $p_{c}$, is a function of $\rho$, a parameter of $Q_{m f}$ which is related to its anisotropy. We investigate the relation between $p_{c}$ and the average number of neighbors of the blocks as well as the anisotropy of $Q_{m f}$.
\end{abstract}

\section{Introduction}

Due to the work of many physicists, and particularly to the contributions of Dietrich Stauffer, percolation theory has became a powerful tool in Science describing phenomena in many areas as geology, biology, magnetism, or social phenomena $[1,2]$. Despite the enormous success of percolation it has been a theory studied in a support (lattice) that has a single dimension. The only references relating percolation and multifractality concern to the multifractal properties of some quantities of the spanning cluster at the percolation threshold $[3,4]$.

Recently, a model to study percolation in a multifractal was proposed [5] in the literature. In fact, the authors have created an original multifractal object, $Q_{m f}$, and an efficient way to estimate its percolation properties. In this work we study in detail the method to estimate $p_{c}$ for this multifractal and discuss the relation between $p_{c}$, some topologic characteristics, and the anisotropy of $Q_{m f}$.

The multifractal object we develop, $Q_{m f}$, is an intuitive generalization of the square lattice [5]. Suppose that in the construction of the square lattice we use the following algorithm: take a square of size 1 and cut it symmetrically with vertical and horizontal lines. Repeat this process $n$-times; at the $n^{t h}$ step we have a regular square lattice with $2^{n} \times 2^{n}$ cells. The setup algorithm of $Q_{m f}$ is quite similar, the main difference is that we do not cut the square in a symmetric way. In section 2 we explain in detail this algorithm.

The development of $Q_{m f}$ has a twofold motivation. Firstly, there are systems like oil reservoirs that show multifractal properties [6] and are good candidates to be modeled by such object. Secondly, there is indeed a much more general scope: we want to study percolation phenomena in lattices that are not regular, but that are multifractal in the geometrical sense. It is important to know how site percolation transition happens in lattices in which the cells vary in size and also in the number of neighbors.

In this work we analyse some geometric and topologic properties of the percolation cluster generated on $Q_{m f}$ at the percolation threshold. The paper is organized as follows: in
Section II we present the process of construction of $Q_{m f}$ and the algorithm for the estimation of $p_{c}$, in Section III we show the numerical simulations concerning the percolation threshold $p_{c}$ and the topologic properties of $Q_{m f}$; and finally in Section IV we present our final remarks and comments.

\section{The model}

In this section we show the process of building the multifractal $Q_{m f}$ and the key concepts to estimate $p_{c}$. We start with a square of linear size 1 and a partition parameter $0<\rho<1$. For reasons that will be clear later, $\rho=\frac{s}{r}$, where $s$ and $r$ are integers. We call the multifractal built from this parameter $\rho$ as the $" \rho-Q_{m f} "$, or $"(r, s)-Q_{m f} "$.

The first step, $n=1$, consists of two sections of the square: a vertical and an horizontal. Initially the square is cut in two pieces of area $\frac{r}{s+r}=\frac{1}{1+\rho}$ and $\frac{s}{s+r}=\frac{\rho}{1+\rho}$ by a vertical line. This process is shown in Fig. 1(a), where we use as an example $\rho=\frac{s}{r}=\frac{2}{3}$. The horizontal cut in which we use the same partition $\rho$ is shown in Fig. 1(b). The first partition of the square generates four rectangular blocks: the largest one of area $\left(\frac{\rho}{1+\rho}\right)^{2}$, two of area $\frac{\rho}{(1+\rho)^{2}}$ and the smallest one of area $\left(\frac{1}{1+\rho}\right)^{2}$. The difference between the largest area and the smallest one increases as $\rho \rightarrow 0$, moreover, the blocks get more and more stretched in this limit. Therefore $\rho$ measures the anisotropy of $Q_{m f}$.

In the second step, $n=2$, we repeat the same process of vertical and horizontal sections as in step 1. Generically we get $2^{2 n}$ blocks after the $n^{t h}$-step. The partition process produces a set of blocks with a variety of areas. We call a set of all elements with the same area as a $k$-set. At the $n^{t h}$-step of the algorithm the partition of the square in blocks follows the binomial rule:

$$
A=\sum_{k=0}^{n} C_{k}^{n}\left(\frac{\rho}{1+\rho}\right)^{k}\left(\frac{1}{1+\rho}\right)^{n-k}=\left(\frac{\rho+1}{\rho+1}\right)^{n}=1 .
$$


(a)

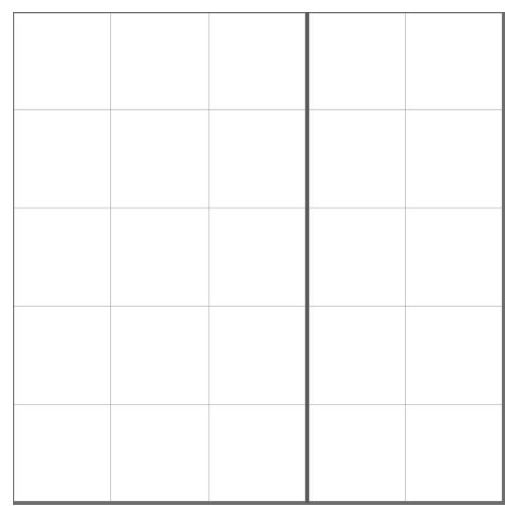

(b)

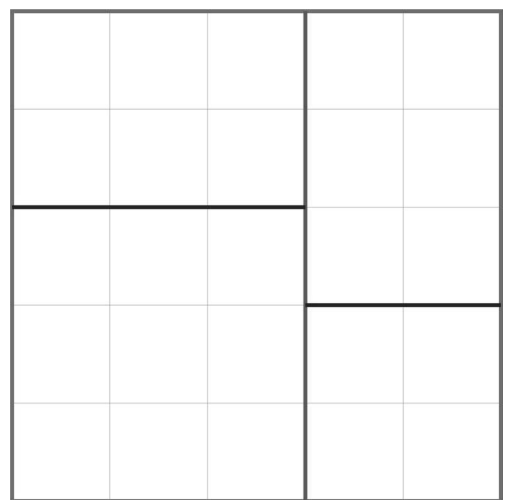

Figure 1. The initial step, $n=1$, in the formation of $Q_{m f}$. In (a) a vertical line cut the square in two pieces according to $\rho$. Two horizontal lines sectioning the rectangles by the same ratio are depicted in (b).. The underlying square lattice is depicted with thin lines.

The number of elements of a $k$-set is $C_{k}^{n}$. In reference [5] we see that as $n \rightarrow \infty$ all the $k$-sets determine a monofractal whose dimension is $d_{k}=\lim _{n \rightarrow \infty} \frac{\log C_{n}^{k} s^{k} r^{(n-k)}}{\log (s+r)^{\frac{n}{2}}}$. In this limit, the ensemble of all $k$-sets engenders the multifractal object $Q_{m f}$ itself.

Figure 2 shows a picture of $Q_{m f}$ for $\rho=\frac{1}{3}$ and $n=4$. We use the following code color: blocks of equal area have the same tonality. In other words, all the blocks of a same $k$-set share a common gray-tone. The general view of the object shows an anisotropic, heterogeneous lattice with a non-trivial topology. The anisotropy of $Q_{m f}$ will change the percolation threshold as we investigate in the next section. Before that we study in more detail the problem of percolation.

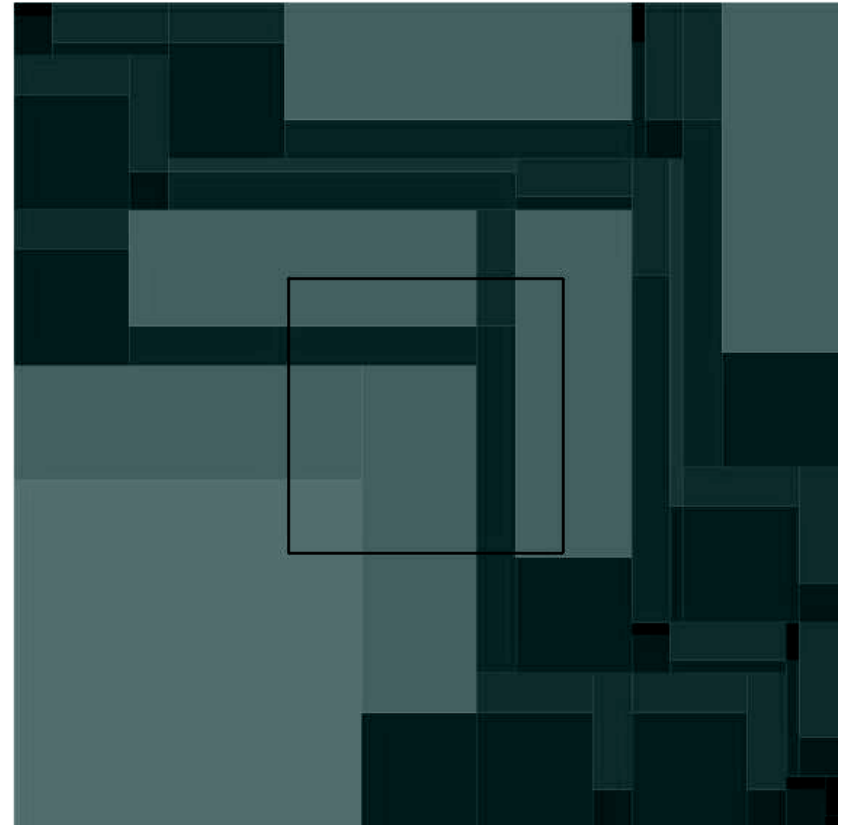

Figure 2. The object $Q_{m f}$ for $\rho=\frac{1}{3}$ and $n=4$, we introduce a square inside the figure to help the visualization.

The main subject of this work is the study of percolation properties of $Q_{m f}$. To perform such a task we develop a percolation algorithm. The percolation algorithm for $Q_{m f}$ start mapping this object into the square lattice. The square lattice should be large enough that each line segment of $Q_{m f}$ coincides with a line of the lattice, this condition imposes that $\rho$ is a rational number (it means, $r$ and $s$ are integers). Therefore we consider that the square lattice is more finely divided than $Q_{m f}$. In this way all blocks of the multifractal are composed by a finite number of cells of the square lattice. To explain the percolation algorithm we suppose that $Q_{m f}$ construction is at step $n$. We proceed the percolation algorithm by choosing at random one among the $2^{2 n}$ blocks of $Q_{m f}$ independent of its size or number of neighbors. Once a block is chosen all the cells in the square lattice corresponding to this block are considered as occupied. Each time a block of $Q_{m f}$ is chosen the algorithm check if the occupied cells at the underlying square lattice are connected in such a way to form a spanning percolation cluster. The algorithm to check percolation is similar to the one used in $[7,8,9,10]$.

\section{Numerical results}

In this section we show numerical results concerning the percolation threshold. Before the numerics we introduce some definitions. We call lattice the square lattice underlying $Q_{m f}$. Following the literature [1] we call $p$ the probability of occupation of a lattice site. $R_{L}$ is the probability that for a site occupation $p$ there exists a contiguous cluster of occupied sites which crosses completely the square lattice of size $L . p_{c}$ is the probability of occupation at the percolation threshold. There are several ways [8] to define 
$R_{L}$. We use two of them: $R_{L}^{e}$ is the probability that there exits a cluster crossing either the horizontal or the vertical direction, and $R_{L}^{b}$ is the probability that there exits a cluster crossing both directions. At the limit of infinite lattice size $R_{L}^{e}$ and $R_{L}^{b}$ converge to a common value for the square lattice case. Besides we call $p_{c}^{e}$ the value of $p_{c}$ estimated from $R_{L}^{e}$, it means, the average $p_{c}$ over lattices that percolate in one direction, the horizontal or the vertical. And, similarly, $p_{c}^{b}$ the value of $p_{c}$ estimated from $R_{L}^{b}$.

(a)

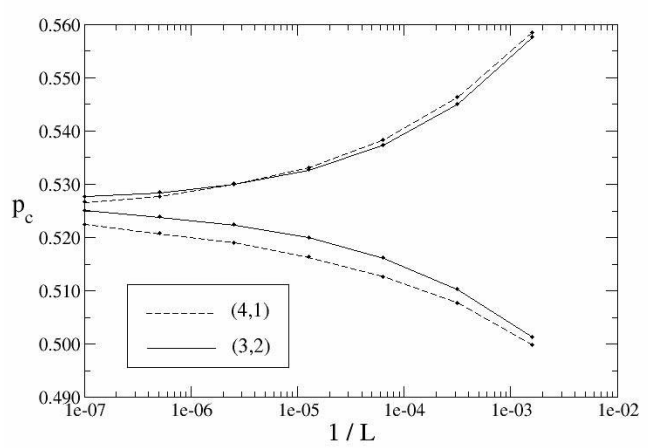

(b)

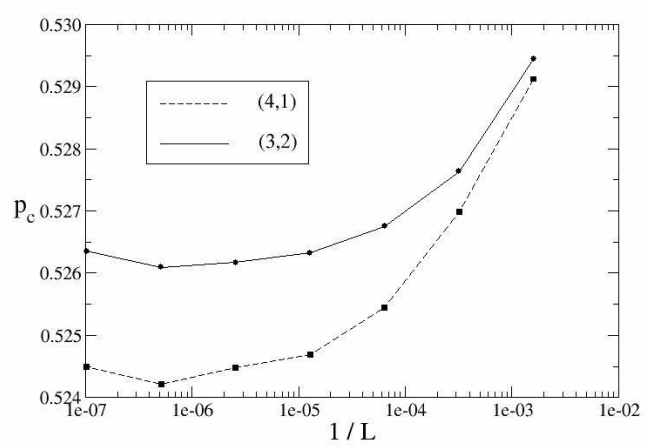

Figure 3. In (a) we show $p_{c}$ versus $\frac{1}{L}$ for $\rho=\frac{2}{3}$ (solid line) and $\rho=\frac{1}{4}$ (dashed line). The upper values correspond to $p_{c}^{b}$ and the lower values to $p_{c}^{e}$; it is used $4 \leq n \leq 10$. In (b) we plot $p_{\text {ave }}$ versus $\frac{1}{L}$ for the same data.

Figure 3 illustrates the behavior of $p_{c}^{e}$ and $p_{c}^{b}$ for 7 different lattice sizes. Two typical values of $\rho$ are used: $\rho=\frac{2}{3}$ (solid line) and $\rho=\frac{1}{4}$ (dashed line). In Fig. 3(a) we show both $p_{c}$ versus $\frac{1}{L}$, the upper values corresponds to $p_{c}^{b}$ and the bottom values to $p_{c}^{e}$. The lattice size, $L=(r+s)^{n}$, corresponds to $4 \leq n \leq 10$, in both cases $r+s=5$. The data of the upper branch of the figure collapse into a single curve, diversly from the lower branch. In other words, the curves of $(2,3)-Q_{m f}$ and $(4,1)-Q_{m f}$ share roughly the same $p_{c}^{e}$ but diverse $p_{c}^{b}$. The reason for this feature is the strong anisotropy of $(4,1)-Q_{m f}$ compared to the case $(2,3)-Q_{m f}$. The anisotropy of the percolation cluster does not affect $p_{c}$ in both directions, but it affects $p_{c}$ in one direction. In fact, the percolation in both directions comes from an average over both directions, in such situation, any eventual anisotropy effect of the percolation cluster vanishes because of the average.

An useful way to define $p_{c}$ is to take the average value $p_{c_{\text {ave }}}=\frac{p_{c}^{e}+p_{c}^{b}}{2}$. In Fig. 3(b) we plot $p_{c_{a v e}}$ versus $\frac{1}{L}$ corresponding to the same data Fig. 3(a). We observe in this figure that both curves converge to a saturation value that is not the same. The difference between the two cases is related to the curve of $p_{c}^{b}$. The anisotropy (stretching of the blocks) due to $\rho$ implies an anisotropy in the percolation cluster. Such anisotropy determines that the percolation cluster does not have a correlation length independent of the direction. Because the percolation cluster is anisotropic the symmetry between $p_{c}^{e}$ and $p_{c}^{b}$ should fail. Therefore, we expect that for smaller $\rho$ (more anisotropy in the multifractal) a greater difference in $p_{c_{a v e}}$ will appear. Fig. 4 confirm this tendency for diverse values of $\rho$.

Figure 4 shows $\bar{p}_{c}$ versus $\rho$. To obtain $\bar{p}_{c}$ we make an average of $p_{c_{a v e}}$ after the saturation process, it means, for $n \geq 8$. The parameter $\rho$ is indicated in the figure. These values are also shown in Table I. The straight line in the figure is the linear fitting of the data. We focus our attention on two features of the figure: the general tendency of decreasing $p_{c}$ with $\rho$, and the anomalous case $(3,1)-Q_{m f}$. The main tendency of decreasing $p_{c}$ with $\rho$ we have discussed in connection with anisotropy. We comment the anomalous situation of $(3,1)-Q_{m f}$ in relation to topologic properties of $Q_{m f}$ which are analyzed in what follows.

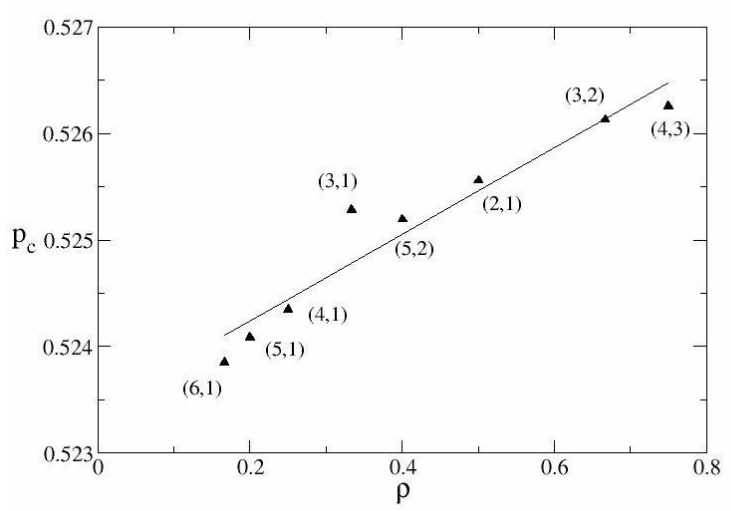

Figure 4. The $\bar{p}_{c}$ versus $\rho$. The chosen values of $\rho$ are indicated in the figure. The straight line corresponds to the linear fitting.

The object $Q_{m f}$ is build of a set of blocks $i$ with different areas, $A_{i}$, and number of neighbors, $\zeta_{i}$. The number $\zeta_{i}$ is a central quantity in the study of topology. A topologic quantity, that is important in the investigation of the percolation threshold, is $\zeta_{\text {ave }}$ which is an average of $\zeta_{i}$ over $Q_{m f}$. It means $\zeta_{\text {ave }}=\frac{\sum_{i} \zeta_{i}}{N}$ where $N=2^{2 n}$ is the total number of blocks and the sum in the numerator is performed over the full multifractal. Typically $\zeta_{\text {ave }}$ is a number that does not increase with $n$, but attains a constant value after $n \approx 6$. We plot in Table I this topologic quantity for $n=8$ and several values of $\rho$. 
Table I

\begin{tabular}{ccccccccc}
\hline$(s, r)$ & $(1,1)$ & $(4,3)$ & $(3,2)$ & $(2,1)$ & $(5,2)$ & $(3,1)$ & $(4,1)$ & $(5,1)$ \\
\hline$p_{c}$ & 0.5929 & 0.5262 & 0.5262 & 0.5256 & 0.5252 & 0.5253 & 0.5243 & 0.5241 \\
$\zeta_{\text {ave }}$ & 999 & 5.436 & 5.436 & 5.434 & 5.436 & 5.426 & 5.436 & 5.436 \\
\hline
\end{tabular}

We observe in the row of $\zeta_{a v e}$ that all these values are roughly the same, the exception corresponds to the case of the $(3,1)-Q_{m f}$. The conclusion we take is that the variation of the average number of neighbors causes the fluctuation in percolation threshold observed in Fig. 4. The $p_{c}$ of the $(3,1)-Q_{m f}$ is a little bit greater than the other cases because it has a smaller average number of neighbors. In other words, because each block of the $(3,1)-Q_{m f}$, in the average, has less neighbors, it percolates with more difficulty than the tendency among its group.

\section{Final remarks}

To summarize we analyze in this work the role of the anisotropy and the average number of neighbors of $Q_{m f}$ on its percolation threshold, $p_{c}$. The multifractal object is composed by a set of blocks with different areas and number of neighbors. As the parameter defining $Q_{m f}, \rho$, goes to zero, the multifractal becomes more and more anisotropic. This anisotropy reflects in the percolation cluster creating an asymmetry between $p_{c}^{e}$ and $p_{c}^{b}$.

The anisotropy of $Q_{m f}$ is evident when we compare $p_{c}^{e}$ and $p_{c}^{b}$. The observed curves of $p_{c}^{e}$ show a similar behavior, in contrast to the curves of $p_{c}^{b}$. Actually, the measure of $p_{c}^{b}$ makes an average over both directions which erases any anisotropic effect of the percolation cluster. This erasing effect does not exist when we measure $p_{c}^{e}$. The anisotropy of $Q_{m f}$ decreases with $\rho$ (with $\rho \rightarrow 0$ the blocks became more stretched).

A special case in our analysis is the $(3,1)-Q_{m f}$. This case is singular compared to others analyzed cases. For a same number of blocks, the $(3,1)-Q_{m f}$ case has less neighbors than the multifractal blocks corresponding to other values of $\rho$. This phenomenon is intrinsic to the topology of $Q_{m f}$. It implies that the $(3,1)-Q_{m f}$ is less connected, and, as a consequence, it shows more difficulty to perco- late. Therefore the case of the $(3,1)-Q_{m f}$ has a percolation threshold slightly higher than its neighbors in the $\rho$ sequence. In a future work we intend to study in more detail the effect of other topologic characteristics on the percolation properties.

The authors gratefully acknowledge the financial support of Conselho Nacional de Desenvolvimento Científico e Tecnológico (CNPq)-Brazil, FINEP and CTPETRO.

\section{References}

[1] D. Stauffer and A. Aharony, Introduction to Percolation Theory, (Taylor \& Francis, London, 1994)

[2] M. Sahimi, Applications of Percolation Theory, (Taylor \& Francis, Bristol, 1994).

[3] K.P.N. Murthy, L.K. Gallos, P. Argyrakis, and K. N. Kehr, Phys. Rev. E, 546922 (1996).

[4] O. Stenull and H. K. Jenssen, Phys. Rev. E, 65045104 (2002).

[5] G. Corso, J. E. Freitas, L. S. Lucena, and R. F. Soares, Phys. Rev. E, in press

[6] F. Herrmann, A Scaling Medium Representation, a Discussion on Well-logs, Fractals and Waves. PhD Thesis, Delft University of Technology, (1997).

[7] J. E. de Freitas, L. S. Lucena, and S. Roux, Physica A 26681 (1999).

[8] M. E. J. Newman and R. M. Ziff, Phys. Rev. Lett. 854104 (2000).

[9] J. E. de Freitas and L. S. Lucena, IJMPC, 111581 (2000).

[10] J. E. de Freitas, L. S. Lucena, and S. Roux, Phys. Rev. E, 64, 051405 (2001). 